\title{
Optimal Beamforming for Multicasting Data in CMHP
}

\author{
Hyung-Tae Kim, Sung-Hoon Lim and Sae-Young Chung \\ Department of Electrical Engineering and Computer Science \\ Korea Advanced Institute of Science and Technology \\ E-mail: kukudas@kaist.ac.kr, cruse77@kaist.ac.kr, sychung@ee.kaist.ac.kr
}

\begin{abstract}
In this paper we show the optimal solution of the beamforming vectors of a multicast setting, in which the transmitter has multiple antennas that sends common data to multiple receivers. We consider two important applications such as the multicasting scenario itself and the use of the common information in a superposition coding scheme known as the Cover [1] - Van der Meulen [2]- Hajek - Pursley's [3] (CMHP[7]) method. We show that the optimization of the beamforming vector is equivalent to a vector projection problem.
\end{abstract}

\section{INTRODUCTION}

$\mathbf{T}$ HE capacity of the the single input single output (SISO) broadcast channel has been known to be achieved by a combination of superposition coding and successive interference cancellation (SIC). This is true for Gaussian channels with the degraded property, where one user has a noisier version of the other users channels [4]. However, the multiple input multiple output (MIMO) downlink channel is a non-degraded channel and this property makes the use of superposition coding with SIC suboptimal. In the case where we have perfect channel state information (CSI) dirty paper coding (DPC) is an optimal strategy that achieves the capacity of the MIMO broadcast channel [5]-[6] and several practical progresses using source-channel coding in the dirty paper channel have been made in this area [8]-[10]. However, there is still some difficulty in practically implementing this scheme due to high complexity. This motivated a search for suboptimal schemes with fair complexity. CMHP is a strong candidate for such need and has been shown to have better performance compared to TDMA, zeroforcing and MMSE.

CMHP also has fair complexity since it requires only beamforming transmitters and SIC receivers. In addition, CMHP performs better than DPC in high SNRs and nearly as well as DPC in low SNRs if only limited CSI is available at the transmitter [11]. Although the simple structure where it only needs beamforming at the transmitter and SIC at the receivers, the optimization for the parameters at the transmitter (e.g. beamforming vectors and power allocation) is not an easy task. As will be explained in the upcoming sections, CMHP makes use of a common information term where it is defined as the data decodable to all users. In [7] the authors use an iterative algorithm that iterates between power optimization and beamforming optimization. As a part of this iteration, we will show the optimal beamforming solution for the common information.

The organization of this paper is as follows. Section II describes the multiuser MISO system model with common data. We will review the CMHP and show our main result in Section III. Finally, Section IV concludes the paper.

\section{System MODEL}

\section{A. Notation}

The vector and matrix are expressed by boldface. $\|\mathrm{x}\|$ is defined as $\|\mathbf{x}\|=\sqrt{x_{1}^{2}+\cdots+x_{K}^{2}}$ and $\operatorname{diag}(\mathbf{x})$ denotes the diagonal matrix with diagonal elemnets as $\mathbf{x} . N_{\mathbb{C}}(\mu, \sigma)$ stands for the circularly symmetric Gaussian random variable with mean and variance of $\mu$ and $\sigma$ respectively.

\section{B. MISO Broadcast channel model}

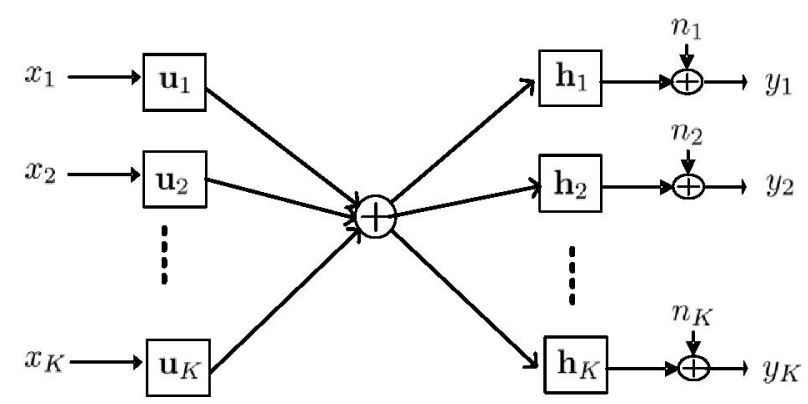

Fig. 1. MISO downlink channel

We consider the Gaussian, complex and memoryless, MISO Broadcast system where a base station with M transmit antennas transmits different signals to multiple mobile users, where each user has one receive antenna. Let $\mathrm{K}$ be the number of users.

To ensure that the users receive their data without coordination, an appropriate preprocessing of the data should be carried out at the transmitter. Let $\mathbf{V}$ denote the linear preprocessing matrix and $x_{k} \in \mathbb{C}$ is the encoded data for the $k$-th user. We assume the encoded data has Gaussian distribution. Then, the transmitted signal is represented by:

$$
\mathbf{s}=\mathbf{V X}_{\mathbf{X}}
$$


where $\mathbf{V}$ is a matrix decomposed as $\mathbf{V}=\mathbf{U P}$ and $\mathbf{s}$ is an $M$-by-1 complex vector. Here $\mathrm{U}$ is the beamforming matrix with column vectors $\mathbf{u}_{i} \in \mathbb{C}^{\mathbb{M}}$ as the beamforming vector for user $i . \mathbf{u}_{i}$ is nomalized so that $\left\|\mathbf{u}_{i}\right\|=1$. $\mathbf{P}$ is defined by $\operatorname{diag}\left(\left[p_{1}, \ldots, p_{K}\right]\right)$ where $p_{i} \in \mathbb{R}$ is the power allocated to the $i t h$ user. We assume The total power constraint is $P_{\max }$. So $\sum_{i} p_{i} \leq P_{\max }$ must be satisfied.

The received signal $y_{k}$ at the $k$-th user, an $M$-by- 1 vector, is represented by

$$
y_{k}=\mathbf{h}_{k} \mathbf{s}+n_{k}, k=1, \ldots, K,
$$

where $\mathbf{h}_{k} \in \mathbb{C}^{\mathbb{M}}$ is a row vector consisting of frequency flat channel realizations between $\mathrm{M}$ transmitter antennas and the $i$ th user antenna for $k=1, \ldots, K . n_{k} \in \mathbb{C}$ is circularly symmetric Gaussian noise $N_{\mathbb{C}}(0,1)$ caused by thermal noise.

\section{Optimal BeAmForming FOR THE CMHP}

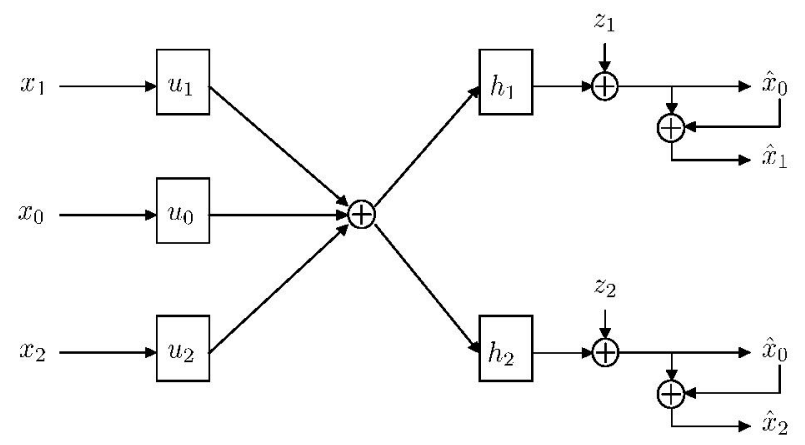

Fig. 2. 2-user CMHP

In this paper we consider the 2-user CMHP with multiple antennas at the transmitter and single antennas at the receivers. The 2-user CMHP rate region is given by the following,

$$
\mathcal{R}^{c m h p}=\operatorname{Co}\left(R^{c m h p}\right)
$$

where $\operatorname{Co}(a)$ is the convex hull operation of $a$,

$$
\begin{aligned}
& R^{c m h p} \triangleq \\
& \bigcup_{\sum_{i} p_{i} \leq P}\left\{\begin{array}{l}
R_{1} \leq \log \left(1+\frac{\left|\mathbf{h}_{1} \mathbf{u}_{1}\right|^{2} p_{1}}{1+\left|\mathbf{h}_{1} \mathbf{u}_{2}\right|^{2} p_{2}}\right) \\
R_{2} \leq \log \left(1+\frac{\left|\mathbf{h}_{2} \mathbf{u}_{2}\right|^{2} p_{2}}{1+\left|\mathbf{h}_{2} \mathbf{u}_{1}\right|^{2} p_{1}}\right) \\
R_{0} \leq \min _{i=1,2} \log \left(1+\frac{\left|\mathbf{h}_{i} \mathbf{u}_{0}\right|^{2} p_{0}}{1+\left|\mathbf{h}_{i} \mathbf{u}_{1}\right|{ }^{2} p_{1}+\left|\mathbf{h}_{i} \mathbf{u}_{2}\right|^{2} p_{2}}\right)
\end{array}\right.
\end{aligned}
$$

$\mathbf{h}_{k}$ is the channel vector, $\mathbf{u}_{k}$ is the beamforming vector, and $p_{k}$ is the power allocated to user $\mathrm{k}$ with total power constraint $P_{\max }$, where $\mathrm{k}=0$ is the index for the common information. $R_{1}, R_{2}$ are the achievable rates of private information for user 1 and user 2 , respectively. $R_{0}$ is the achievable rate of the common information that user 1 and user 2 can both decode. We ensure this by taking the minimum of $R_{0}$ sent to user 1 or user 2 . Notice that the common information does not necessarily hold common data for both users. Since the common information can be decoded at both user 1 and user 2, not only can we send data intended for both users, we can use the common information term to send private information to user 1 or user 2 . The user that is assigned the private information should just decode $R_{0}$, and the user that does not have his data assigned decodes $R_{0}$ and does successive interference cancellation. This will enable the unassigned user to increase its SINR that is free of the interference caused from the common information. For each user we will pick the optimal powers and beamforming vectors that maximizes the weighted sum rate. For the process of optimizing the beamforming vectors, we make use of the transformation results in [12]. The duality result in [12] tells us that if the sum power constraints are the same, using the same beamforming vectors for the original broadcast channel and the dual MAC channel, we can have the same set of SINR's that results in the same rate in both domains. We use this result to convert the original $\mathrm{BC}$ beamforming problem to its dual MAC problem. We do this since the process of finding the optimal beamforming vectors in the $\mathrm{BC}$ domain is more complicated than finding it in the MAC domain. This is because the optimal beamforming for MAC channels are known to be the MMSE receiver beamforming.

In the CMHP case, however, because common information is defined as

$$
\min _{i=1,2} \log \left(1+\frac{\left|\mathbf{h}_{i} \mathbf{u}_{0}\right|^{2} p_{0}}{1+\left|\mathbf{h}_{i} \mathbf{u}_{1}\right|^{2} p_{1}+\left|\mathbf{h}_{i} \mathbf{u}_{2}\right|^{2} p_{2}}\right)
$$

general BC-MAC duality is not satisfied. In [7], the results of the BC-MAC duality has been extended to hold for any general interference cancellation scheme. For CMHP, however, we need to extend the original broadcast region by treating the two rates inside the min() operation as individual users. We will index these extended virtual users as user 3 and 4 . The extended rate region is shown as follows.

$$
R^{\text {extend }} \triangleq
$$

$$
\left\{\begin{array}{l}
R_{1} \leq \log \left(1+\frac{\left|\mathbf{h}_{1} \mathbf{u}_{1}\right|^{2} p_{1}}{1+\left|\mathbf{h}_{1} \mathbf{u}_{2}\right|^{2} p_{2}}\right) \\
R_{2} \leq \log \left(1+\frac{\left|\mathbf{h}_{2} \mathbf{u}_{2}\right|^{2} p_{2}}{1+\left|\mathbf{h}_{2} \mathbf{u}_{1}\right|^{2} p_{1}}\right) \\
R_{3} \leq \log \left(1+\frac{\left|\mathbf{h}_{1} \mathbf{u}_{3}\right|^{2} p_{0}}{1+\left|\mathbf{h}_{1} \mathbf{u}_{1}\right|^{2} p_{1}+\left|\mathbf{h}_{1} \mathbf{u}_{2}\right|^{2} p_{2}}\right) \\
R_{4} \leq \log \left(1+\frac{\left|\mathbf{h}_{2} \mathbf{u}_{4}\right|^{2} p_{0}}{1+\left|\mathbf{h}_{2} \mathbf{u}_{1}\right|^{2} p_{1}+\left.\mathbf{h}_{2} \mathbf{u}_{2}\right|^{2} p_{2}}\right),
\end{array}\right.
$$

where $u_{3}$ and $u_{4}$ are beamforming vectors for virtual user3 and 4 , respectively. Since they are actually the common stream, $u_{3}=u_{4}=u_{0}$. Although the original CMHP problem does not have a dual MAC channel, this extended version does have the dual MAC except the fact that the sum power constraint becomes $p_{1}+p_{2}+p_{0}+p_{0}=q_{1}+q_{2}+q_{3}+q_{4}$ rather than $p_{1}+p_{2}+p_{0}=P_{\max } . q_{1}, q_{2}, q_{3}, q_{4}$ are power of user 1,2 and virtual user 3,4 in the MAC domain, respectively. The rate region of the dual MAC of this extended channel is given by,

$$
R^{\text {extend_MAC }} \triangleq
$$




$$
\left\{\begin{array}{l}
R_{1} \leq \log \left(1+\frac{\mid \mathbf{h}_{1} \mathbf{u}_{1}{ }^{2} q_{1}}{1+\left|\mathbf{h}_{2} \mathbf{u}_{1}\right|^{2} q_{2}+\left.\left|\mathbf{h}_{1} \mathbf{u}_{1}{ }^{2} q_{3}+\right| \mathbf{h}_{2} \mathbf{u}_{1}\right|^{2} q_{4}}\right) \\
R_{2} \leq \log \left(1+\frac{\left|\mathbf{h}_{2} \mathbf{u}_{2}\right|^{2} q_{2}}{1+\left|\mathbf{h}_{1} \mathbf{u}_{2}\right|^{2} q_{1}+\left|\mathbf{h}_{1} \mathbf{u}_{2}\right|^{2} q_{3}+\left|\mathbf{h}_{2} \mathbf{u}_{2}\right|{ }^{2} q_{4}}\right) \\
R_{3} \leq \log \left(1+\left|\mathbf{h}_{1} \mathbf{u}_{3}\right|^{2} q_{3}\right) \\
R_{4} \leq \log \left(1+\left|\mathbf{h}_{2} \mathbf{u}_{4}\right|^{2} q_{4}\right)
\end{array}\right.
$$

and the MAC domain power constraint becomes

$$
p_{1}+p_{2}+p_{0}+p_{0}=q_{1}+q_{2}+q_{3}+q_{4} .
$$

and

$$
p_{1}+p_{2}+p_{0} \leq P \max .
$$

To maximize the minimum of $R_{3}$ and $R_{4}$ we use the following theorem.

Theorem 1(beamforming solution for the common information): The solution to the finding the optimal beamform vector $\mathbf{u}_{0}$ can be divided into three cases and is given as follows when we assume the channel is real and $2 \times 1$ MISO.

1) if $\mathbf{h}_{2} \mathbf{h}_{1}^{*} \sqrt{q_{4}}>\left\|\mathbf{h}_{1}\right\|^{2} \sqrt{q_{3}}$

Then the solution to $\mathbf{u}_{0}$ is $\mathbf{u}_{0}=\frac{\mathbf{h}_{1}^{*}}{\left\|\mathbf{h}_{1}\right\|}$

This accounts for the case where even if we align the beamforming vector in favor of user 3 we will still have user 3 as the minimum of the two virtual users.

2) $\mathbf{h}_{1} \mathbf{h}_{2}^{*} \sqrt{q_{3}}>\left\|\mathbf{h}_{2}\right\|^{2} \sqrt{q_{4}}$

Then the solution to $\mathbf{u}_{0}$ is

$$
\mathbf{u}_{0}=\frac{\mathbf{h}_{2}^{*}}{\left\|\mathbf{h}_{2}\right\|}
$$

This accounts for the case where even if aligning the beamforming vector in favor of user 4 we still would have user 4 as the minimum of the two virtual users.

3) Else

a) $\mathbf{h}_{\mathbf{1}} \cdot \mathbf{h}_{\mathbf{2}}<0$

Then the solution to $\mathbf{u}_{0}$ is $\mathbf{u}_{0}=\left(\mathbf{h}_{1} \sqrt{q_{3}}+\mathbf{h}_{2} \sqrt{q_{4}}\right)^{\perp}$

b) $\mathbf{h}_{\mathbf{1}} \cdot \mathbf{h}_{\mathbf{2}} \geq 0$

Then the solution to $\mathbf{u}_{0}$ is

$$
\mathbf{u}_{0}=\left(\mathbf{h}_{1} \sqrt{q_{3}}-\mathbf{h}_{2} \sqrt{q_{4}}\right)^{\perp}
$$

Where the $\mathbf{a}^{\perp}$ operation gives a unit-norm vector that is perpendicular to $\mathbf{a}$. This case accounts for the case where we have to balance the rates of user 3 and user4 to maximize the minimum rate.

proof: Our problem is to find optimal $u_{0}$ as follows:

$$
\max _{u_{0}} \min \left(\left|\mathbf{h}_{1}^{\prime} \cdot \mathbf{u}_{0}\right|,\left|\mathbf{h}_{2}^{\prime} \cdot \mathbf{u}_{0}\right|\right) .
$$

For any $\mathbf{h}_{1}^{\prime}, \mathbf{h}_{2}^{\prime}$ which is called effective channel, we can divide the effective channel conditions into three distinct

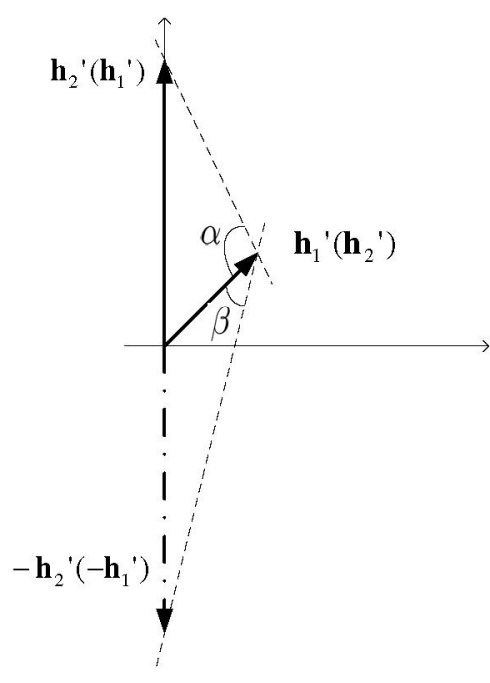

(a)

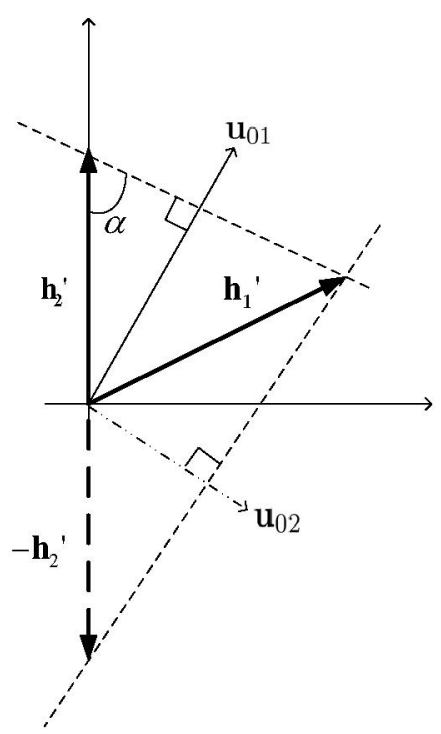

(b)

Fig. 3. The channel vectors for the cases in Theorem $1, \mathbf{h}_{1}^{\prime}=$ $\mathbf{h}_{1}^{\mathrm{T}} \sqrt{q_{3}}, \mathbf{h}_{2}^{\prime}=\mathbf{h}_{2}^{\mathrm{T}} \sqrt{q_{4}}$ (a) channel for case 1 and 2 (b) channel for case 3

cases,

$$
\begin{aligned}
& \left|\mathbf{h}_{\mathbf{1}}^{\prime} \cdot \frac{\mathbf{h}_{1}^{\prime}}{\left\|\mathbf{h}_{1}^{\prime}\right\|}\right|<\left|\mathbf{h}_{\mathbf{2}}^{\prime} \cdot \frac{\mathbf{h}_{1}^{\prime}}{\left\|\mathbf{h}_{1}^{\prime}\right\|}\right| \\
& \left|\mathbf{h}_{\mathbf{1}}^{\prime} \cdot \frac{\mathbf{h}_{\mathbf{2}}^{\prime}}{\left\|\mathbf{h}_{2}^{\prime}\right\|}\right|>\left|\mathbf{h}_{\mathbf{2}}^{\prime} \cdot \frac{\mathbf{h}_{2}^{\prime}}{\left\|\mathbf{h}_{2}^{\prime}\right\|}\right|
\end{aligned}
$$

where $\mathbf{h}_{1}^{\prime}=\mathbf{h}_{1}^{\mathrm{T}} \sqrt{q_{3}}$ and $\mathbf{h}_{2}^{\prime}=\mathbf{h}_{2}^{\mathrm{T}} \sqrt{q_{4}}$. These are the same three categories that we divided in Theorem 1. In case 1 , $\mathbf{u}_{0}$ is $\frac{\mathbf{h}_{1}^{\prime}}{\left\|\mathbf{h}_{1}^{\prime}\right\|}$, because for any other $\mathbf{u}_{0}, \mathbf{h}_{\mathbf{1}}^{\prime} \cdot \mathbf{u}_{0}$ is less than $\mathbf{h}_{1}^{\prime} \cdot \frac{\mathbf{h}_{1}^{\prime}}{\left.\| \mathbf{h}_{1}^{\prime}\right\rceil}$. Similarly, $\mathbf{u}_{0}$ is $\frac{\mathbf{h}_{2}^{\prime}}{\left\|\mathbf{h}_{2}^{\prime}\right\|}$ for case 2 . As for the third case, to maximize the minimum of $\left(\left|\mathbf{h}_{1}^{\prime} \cdot \mathbf{u}_{0}\right|,\left|\mathbf{h}_{2}^{\prime} \cdot \mathbf{u}_{0}\right|\right)$ the projection vector $\mathbf{u}_{0}$ must be the one that makes $\left|\mathbf{h}_{1}^{\prime} \cdot \mathbf{u}_{0}\right|$ 
and $\left|\mathbf{h}_{2}^{\prime} \cdot \mathbf{u}_{0}\right|$ the same. There are two vectors that satisfy this condition. The first vector that is denoted by $\mathbf{u}_{01}$ is $\left(\mathbf{h}_{1} \sqrt{q_{3}}+\mathbf{h}_{2} \sqrt{q_{4}}\right)^{\perp}$ and the second vector denoted by $\mathbf{u}_{02}$ is null $\left(\mathbf{h}_{1} \sqrt{q_{3}}-\mathbf{h}_{2} \sqrt{q_{4}}\right)^{\perp}$. $\mathbf{u}_{0}$ is one of the two vectors that maximizes $\left|\mathbf{h}_{\mathbf{1}}^{\prime} \cdot \mathbf{u}_{\mathbf{0}}\right|$ and $\left|\mathbf{h}_{\mathbf{2}}^{\prime} \cdot \mathbf{u}_{\mathbf{0}}\right|$. We can easily find which one is the solution by considering the channel realization. The graphical representation help you understand the above answers. In Figure 3-a, the first case shows that the angle between $\mathbf{h}_{1}^{\prime}$ and the one of two dotted lines which represents $\mathbf{h}_{1}^{\prime}-\mathbf{h}_{2}^{\prime}$ and $\mathbf{h}_{1}^{\prime}+\mathbf{h}_{2}^{\prime}$ is more than 90 degrees. The reason why we also consider $\mathbf{h}_{1}^{\prime}+\mathbf{h}_{2}^{\prime}$ is that the absolute value of the projection is compared not the signed value. In this case we determine the direction of the projection vector $\mathbf{u}_{0}$ to be the same as $\mathbf{h}_{1}^{\prime}$. We can find the same representation for the second case analogously just by exchanging the index of the effective channel. The first case and second case are distinct because if one angle of the triangle is more than 90 degrees, then the other angle must be less than 90 degrees. The third case happens if the angles between the dotted line and each effective channel is equal or less than 90 degrees. This can be further divided into two subcases denoted by (3-a) and (3-b) in Theorem 1. (3-a) concerns the case where the angle between $\mathbf{h}_{1}^{\prime}$ and $\mathbf{h}_{2}^{\prime}$ is more than 90 degrees, and the solution for this case is $\mathbf{u}_{02}$. This is because the absolute value of the projection of the effective channel on $\mathbf{u}_{02}$ is always larger than the absolute value of projecting the effective channel on $\mathbf{u}_{01}$ in this case. Similarly, the solution is $\mathbf{u}_{01}$ for the (3-b).

The 3 cases are disjoint and covers all possible channel instances. We can also see that each of the 3 optimal solutions of $\mathbf{u}_{0}$ corresponds one of the three cases one to one. Moreover, in the extended system one of the following must be satisfied for the optimal powers and beamforming.

$$
\begin{aligned}
& R_{3}<R_{4} \\
& R_{3}>R_{4} \\
& R_{3}=R_{4}
\end{aligned}
$$

When $R_{3}<R_{4}$ is satisfied, the only possible $\mathbf{u}_{0}$ is one of three case, in exact $\mathbf{u}_{0}$ is $\frac{\mathbf{h}_{1}^{\prime}}{\left\|\mathbf{h}_{1}^{\prime}\right\|}$. When $R_{3}=R_{4}$ or $R_{3}>R_{4}$ is satisfied, $\mathbf{u}_{0}$ is also determined in the same context. We can find the following relation between these two.

$$
\begin{aligned}
& R_{3}<R_{4} \longleftrightarrow u_{0}=\frac{\mathbf{h}_{1}^{\prime}}{\left\|\mathbf{h}_{1}^{\prime}\right\|} \\
& R_{3}>R_{4} \quad \longleftrightarrow u_{0}=\frac{\mathbf{h}_{2}^{\prime}}{\left\|\mathbf{h}_{2}^{\prime}\right\|} \\
& R_{3}=R_{4} \quad \longleftrightarrow u_{0}=\left(\mathbf{h}_{1}^{\prime} \pm \mathbf{h}_{2}^{\prime}\right)^{\perp}
\end{aligned}
$$

We can easily expand the solution for finding the optimal beamforming vector in the complex domain. Considering the complex channel, both $R_{3}<R_{4}$ and $R_{4}<R_{3}$ cases have exactly the same solution as the real channel situation. However for case $3, R_{4}=R_{3}$, the real domain solution does not give the optimal solution for the complex domain. There is a different approach to get the optimal beamforming vector by using the eigenvalue decomposition. The following is the equivalent model for finding the optimal solution for complex channels for the third case in theorem 1.

$$
\begin{aligned}
S I N R_{3} & =S I N R_{4} \\
\left|\mathbf{h}_{\mathbf{1}} \mathbf{u}_{0}\right|^{2} q_{3} & =\left|\mathbf{h}_{\mathbf{2}} \mathbf{u}_{\mathbf{0}}\right|^{2} q_{4} \\
\left|\mathbf{a} \mathbf{u}_{0}\right|^{2} & =\left|\mathbf{b} \mathbf{u}_{\mathbf{0}}\right|^{2} \\
\mathbf{u}_{\mathbf{0}}^{*}\left(\mathbf{a}^{*} \mathbf{a}-\mathbf{b}^{*} \mathbf{b}\right) \mathbf{u}_{0} & =0 \\
\mathbf{u}_{\mathbf{0}}^{*} \mathbf{V} \mathbf{\Lambda} \mathbf{V}^{*} \mathbf{u}_{0} & =0 \\
\mathbf{x}^{*} \mathbf{\Lambda} \mathbf{x} & =0 \\
\lambda_{1}\left|x_{1}\right|^{2}+\lambda_{2}\left|x_{2}\right|^{2} & =0
\end{aligned}
$$

where $\mathbf{a}=\mathbf{h}_{\mathbf{1}} \sqrt{q_{3}}, \mathbf{b}=\mathbf{h}_{\mathbf{2}} \sqrt{q_{4}}, \mathbf{V} \boldsymbol{\Lambda} \mathbf{V}^{*}$ is the singular value decomposition of $\left(\mathbf{a}^{*} \mathbf{a}-\mathbf{b}^{*} \mathbf{b}\right)$, where $\mathbf{V}$ is unitary and $\boldsymbol{\Lambda}=\operatorname{diag}\left[\lambda_{1}, \lambda_{2}\right]$ and $\mathbf{x}=\mathbf{V}^{*} \mathbf{u}_{0}$ whose $i$-th element denoted by $x_{i}$. From the last equation, we can find the optimal beamforming vector that satisfies $R_{4}=R_{3}$ and maximizes $R_{4}$ and $R_{3}$. Q.E.D.

The following is a plot of the 3 dimensional rate region found by using Theorem 1 . We assume that $2 \times 1$ MISO, 2user, $\mathrm{SNR}=10 \mathrm{~dB}$ and the channel matrix is $\left[\begin{array}{cc}1 & 0.4 \\ 0.4 & 1\end{array}\right]$

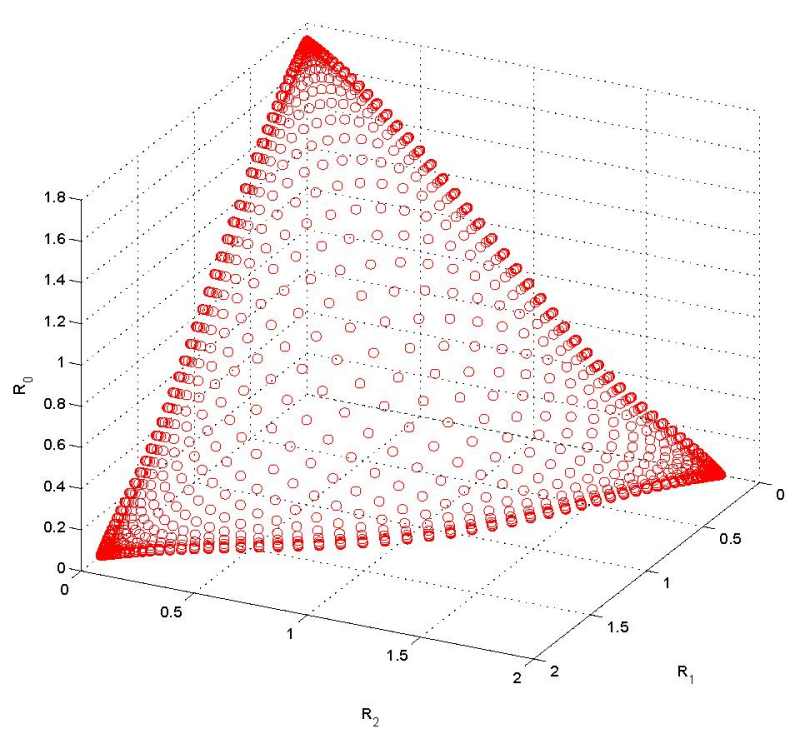

Fig. 4. CMHP 3 dimension rate region

If we set the powers of $p_{1}$ and $p_{2}$ to zero, we only have common information. This is just a multicasting scenario and we can have the solution to this problem with the same results from above by just setting the powers as,

$$
\begin{aligned}
& p_{1}=0 \\
& p_{2}=0 \\
& p_{0}=P_{\max }
\end{aligned}
$$

\section{CONCLUSION}

In this paper we have shown the solution of the beamforming for the common information of the CMHP. This solution works regardless of how we plan to use the common information, whether we use it for common data to both users or private message to one user and the other user does SIC to 
enhance his SINR. The solution is divided into three cases. The three cases actually accounts for the case where the min operator of the common information rate gives the solution for the case of, $R 3>R 4, R 3<R 4$, and $R 3=R 4$. We presented beamforming vectors for all three cases. Although we presented the solution for the 2-user CMHP, it can be easily extended to the $\mathrm{n}$ user cases.

\section{ACKNOWLEDGEMENT}

This work was supported by the Basic Research Program of the Korea Science and Engineering Foundation (R012006-000-11112-0) and it was supported by the center for Broadband OFDM Mobile Access (BrOMA) at POSTECH through the ITRC program of the Korean MIC, supervised by IITA. (IITA-2006-C1090-0603-0037)

\section{REFERENCES}

[1] T. Cover, "An achievable rate region for the broadcast channel," proc. IEEE Trans. on Information Theory, vol.21, no.4, pp.399404, Jul. 1975

[2] E. V. der Meulen, "Random coding theorems for the general discrete memoryless broadcast channel," proc. IEEE Trans. on Information Theory, vol.21, no.2, pp.180-190, Mar. 1975

[3] B. Hajek and M. Pursley, "Evaluation of an achievable rate region for teh broadcast channel," proc. IEEE Trans. on Information Theory, vol.25, no.1, pp.36-46, Jan. 1979

[4] T. M. Cover and J. A. Thomas, "Elements of Information Theory.", New York: Wiley, 1991.

[5] G. Caire and S. S. Shamai, "On the achievable throughput of a multiantenna Gaussian broadcast channel", Information Theory, IEEE Transactions on, vol. 49, pp. 1691-1706, July 2003.

[6] H. Weingarten, Y. Steinberg, and S. Shamai (Shitz), "The capacity region of the Gaussian Multiple Input Multiple Output broadcast channel," in IEEE Trans. on Information Theory,vol.52, no.9, pp. 3936-3964, Sep. 2006

[7] D. Wajeer, A. Wiesel and S. Shamai (Shitz), "Optimal beamforming for the Multi-antenna Gaussian broadcast channel using Superposition coding," in preperation

[8] U. Erez and S. ten Brink, "A close-to-capacity dirty paper coding scheme," IEEE Trans. Inform. Theory, vol. 51, pp. 34173432, Oct. 2005.

[9] Y. Sun, A. D. Liveris, V. Stanković, and Z. Xiong, "Nearcapacity dirty-paper code designs based on TCQ and IRA codes," in Proc. IEEE Int. Symp. Information Theory (ISIT), Sept. 2005, pp. $184-188$.

[10] Y-S. Lee and S.-Y. Chung, "Analysis and Design of Dirty Paper Coding by Transformation of Noise," in submitted to VTC 2007, Sept. 2005, pp. 184-188.

[11] S.-H. Lim and S.-Y. Chung, "Hybrid Dirty Paper Coding and Successive Interference Cancellation for MIMO Downlink Channels," in submitted to comm. letters

[12] P. Viswanath and D. N. C. Tse, "Sum capacity of the vector Gaussian broadcast channel and uplink.downlink duality," IEEE Trans. Inform. Theory, vol. 49, no. 8, pp. 1912.1921, Aug. 2003. 